Article

\title{
Broad-Spectrum Cephalosporin-Resistant and/or Fluoroquinolone-Resistant Enterobacterales Associated with Canine and Feline Urogenital Infections
}

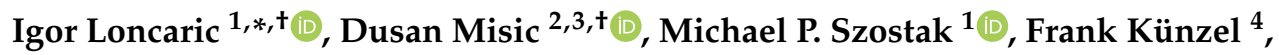 \\ Sabine Schäfer-Somi ${ }^{5}$ and Joachim Spergser ${ }^{1}$ \\ 1 Institute of Microbiology, University of Veterinary Medicine, 1210 Vienna, Austria; \\ michael.szostak@vetmeduni.ac.at (M.P.S.); joachim.spergser@vetmeduni.ac.at (J.S.) \\ 2 Faculty of Biotechnology and Food Science, Wroclaw University of Environmental and Life Sciences, \\ 51-630 Wrocław, Poland; dusan@vet.bg.ac.rs \\ 3 Faculty of Veterinary Medicine, University of Belgrade, 11000 Belgrade, Serbia \\ 4 Clinic for Small Animals, Internal Medicine Unit, University of Veterinary Medicine, 1210 Vienna, Austria; \\ frank.kuenzel@vetmeduni.ac.at \\ 5 Department for Small Animals and Horses, Platform for AI and ET, University of Veterinary Medicine, \\ 1210 Vienna, Austria; sabine.schaefer@vetmeduni.ac.at \\ * Correspondence: igor.loncaric@vetmeduni.ac.at; Tel.: +431250772115 \\ + These authors contributed equally to this work.
}

Received: 28 May 2020; Accepted: 3 July 2020; Published: 7 July 2020

\begin{abstract}
The aim of the present study was to characterize Enterobacterales resistant to 3rd and 4th generation cephalosporins, carbapenems and/or fluoroquinolones, isolated from dogs and cats with urogenital infections. In total, 36 strains (Escherichia coli $(n=28)$, Klebsiella pneumoniae $(n=3)$, Serratia marcescens, Raoultella ornithinolytica, Proteus mirabilis, Citrobacter portucalensis and Enterobacter cloacae (each $n=1)$ ) were included in the present study, 28 from Austria and 8 from Serbia. Isolates were characterized by a polyphasic approach including susceptibility pheno- and genotyping and microarray-based assays. Escherichia (E.) coli isolates were additionally characterized by two-locus (fum $\mathrm{C}$ and fim $\mathrm{H}$ ) sequence phylotyping and multi-locus sequence typing (MLST) of selected isolates. MLST of carbapenem-resistant Enterobacter cloacae isolates was also performed. Among E. coli, the most dominant phylogenetic group was B1 (27.8\%), followed by C, (16.6\%), A and Clade II (5.5\% each), B2 and F (2.77\% each). The most predominant $\beta$-lactam resistance genes were $b l a_{\mathrm{TEM}}(70 \%)$ and $b l a_{\mathrm{CTX}-\mathrm{M}}(38.8 \%), b l a_{\mathrm{CMY}}(25 \%)$. bla $a_{\mathrm{NDM}}$ was detected in one carbapenem-resistant Enterobacter cloacae ST114. The most common ST among selected E. coli was 744 (10.7\% isolates). The pandemic clones ST131 and ST648 carrying CTX-M-15 were also detected. Remaining STs belonged to 469, 1287, 1463 and 1642. E. coli clonotyping revealed $20 \mathrm{CH}$ types. Based on the presence of certain virulence genes, three isolates were categorized as ExPEC/UPEC. The most prevalent virulence factors were fim $\mathrm{H}$ detected in $61 \%$, iucD and iss both in $55 \%$, iro $\mathrm{N}$ in $27.8 \%$, papC in $13.8 \%$ and sat in $8.3 \%$ isolates.
\end{abstract}

Keywords: urogenital system; infection; cephalosporins; fluoroquinolones; Enterobacterales; ESBL; AmpC; carbapenem-resistant; pandemic clone; ST131; ST648; ST114

\section{Introduction}

The most common causative agents of urogenital infections (UGI) are the members of the order Enterobacterales, amongst them primarily Escherichia (E.) coli with a prevalence in companion animals between 35-70\% [1] and in humans between 75-95\% [2]. UGI include infections of the bladder, kidneys 
and urethra and in adult animals also parts of the genital tract such as the uterus in females and prostate in males [3]. E. coli capable of causing UGI is termed uropathogenic E. coli (UPEC) [1,4]. UPECs belong to extraintestinal pathogenic E. coli (ExPEC), which are phylogenetically and epidemiologically distinct from commensal E. coli due to a characteristic combination of virulence factors [1,5-7]. The ExPEC category should simplify the highly complicated categorization of non-intestinal pathogenic E. coli and encompass numerous subcategories which besides UPECs include sepsis-associated E. coli (SEPEC), neonatal meningitis-associated E. coli (NEMEC) and avian-pathogenic E. coli (APEC) $[1,8]$. UGIs can also be caused by DAECs (diffusely adhering E. coli) which in parallel belong to both the extraintestinal and intestinal pathogenic E. coli categories [9]. Opportunistic intestinal E. coli and other intestinal Enterobacterales, which typically lack specific virulence determinants may also cause UGIs [10]. Transmission of UPECs via meat to the human urinary tract has been described and therefore, "foodborne uropathogens" that cause "foodborne urinary tract infections" or FUTI have also been introduced $[1,2,11]$. It has been shown that the above-mentioned ExPEC subcategories mostly share the same virulence factors. They may originate from the human intestinal tract, the environment, livestock, meat products, sewage and companion animals and can cross host species barriers and cause different types of zoonotic infections including UGIs [1,8,10-13]. Typical representatives are E. coli B2-O25b:H4-ST131 and E. coli F-ST648 [2,5,6,10-12,14-16]. Also, the categorization presented here is mostly related to the pathotypes and the origin of isolation, a specific molecular marker for the precise determination of every subcategory is still not defined [17].

Antibiotics are in addition to the uptake of sufficient water essential for the treatment of UGIs [2]. Unfortunately, therapy of UGIs is known to be initiated by the empirical selection of antibiotic agents before microbiological examinations are completed [6,18-20]. For the selection of antibiotics, recommendations made by competent institutions and bodies should be followed. For the treatment of noncomplicated UGI, the International Society for Companion Animal Infectious Disease Committee (ISCAID) recommends amoxicillin or trimethoprim-sulfonamide as first-choice antibiotics, emphasizing that amoxicillin-clavulanic acid should only be the second choice or avoided if possible [18]. All recommendations discourage veterinarians from using fluoroquinolones or 3rd and 4 th generation cephalosporins as first-line antibiotics except if there is a clear indication. The increasing resistance to fluoroquinolones and $\beta$-lactam antibiotics in Enterobacterales with the dominance of CMY- and CTX-M-group harboring strains [2,20-24] but also to so-called "traditional" antibiotics such as sulfamethoxazole-trimethoprim and tetracyclines, somehow renders the recommendations given, limits therapeutic choices and urges clinicians to seek for broader spectrum agents for the treatment of UGIs. In the European Union, including Austria, especially at the University of Veterinary Medicine, Vienna, veterinarians are advised to follow strict guidelines for the use of antibiotics. Except for few countries including the Netherlands and France, veterinarians are allowed to use human-related antibiotics in companion animals, even carbapenems, but only in strictly defined and justified cases [25-28]. Despite carbapenems have no legal indication in some countries and are not used in routine practice, ISCAID and other relevant institutions have included carbapenems in their official documentation related to animals [18,29]. Close contact between humans and companion animals contributes to the propagation and exchange of resistance genes between bacteria as well as the direct transmission of all ExPEC subcategories including UPECs, so human-related clones have already been detected in companion animals [27,30-32]. As a consequence, companion animals are more and more considered an important source of human infections both by ExPEC/UPEC and by multidrug-resistant bacteria (MDR) in general [20,26,33-36].

In the present study, we have characterized a collection of canine and feline Enterobacterales associated with UGI, which were resistant to critically important antibiotics for humans (i.e., 3rd and 4th generations cephalosporins, carbapenems and/or fluoroquinolones) by a multiphasic approach. 


\section{Results}

In Austria, isolates belonging to the order Enterobacterales were detected in 550 of 2398 samples resulting in a carriage rate of $22.94 \%$ whereas 28 isolates were broad-spectrum cephalosporin-resistant and/or fluoroquinolone-resistant resulting in a carriage rate of $1.17 \%$ over all tested animals. We observed higher carriage rates in Serbia. In 45 out of 144 samples (carriage rate 31.25\%), isolates belonging to Enterobacterales were detected whereas eight were broad-spectrum cephalosporin-resistant and/or fluoroquinolone-resistant resulting in a carriage rate of $5.56 \%$ over all tested animals. Twenty-eight isolates were identified as E. coli and the remaining isolates were Klebsiella pneumoniae $(n=3)$, Raoultella ornithinolytica $(n=1)$, Citrobacter portucalensis $(n=1)$, Proteus mirabilis $(n=1)$, Serratia marcescens $(n=1)$ and Enterobacter cloacae $(n=1)$ (Tables 1 and 2$)$. All isolates were resistant to at least one antibiotic from at least three different classes and accordingly, were categorized as MDR [37]. They originated from dogs $(n=30)$ and cats $(n=6)$, wherefrom $58.3 \%(n=21)$ were isolated from urine (obtained via cystocentesis, which is routine), $27.8 \%(n=10)$ from the vagina, $2,7 \%(n=1)$ from an ejaculate, $5.5 \%(n=2)$ from the prostate gland and $5.5 \%(n=2)$ from the bladder wall.

All isolates examined were susceptible to amikacin and only one (Enterobacter cloacae) was resistant to meropenem. Low resistance to nitrofurantoin and fosfomycin (in one Klebsiella pneumoniae and Enterobacter cloacae isolate) was observed. In fourteen isolates (E. coli $(n=11)$, Klebsiella pneumoniae $(n=1)$, Citrobacter portucalensis $(n=1)$ and Enterobacter cloacae $(n=1))$, genes from the bla $a_{\mathrm{CTX}-\mathrm{M}}$ family were detected, alone or in combination with other bla genes. In nine E. coli isolates exhibiting the AmpC/ESBL phenotype, $b l a_{\mathrm{CMY}}$ alone or together with mutation events in the ampC promoter/attenuator region were found. In two isolates displaying the AmpC phenotype, these mutations were detected without the presence of $b l a_{\mathrm{CMY}}$ or other bla genes (Tables 1 and 2$)$. New Delhi metallo- $\beta$-lactamase $\left(b l a_{\mathrm{NDM}-1}\right)$ was found in one carbapenem-resistant Enterobacter cloacae from Serbia. The same isolate harbored $b l a_{\mathrm{CTX}-\mathrm{M}-15}, b l a_{\mathrm{NDM}}$ and bla $a_{\mathrm{OXA}-1}$. Ciprofloxacin resistance was found in $86.1 \%(n=31)$ isolates. Plasmid mediated quinolone resistance (PMQR) genes were identified in 33.3\% $(n=12)$ isolates, of which nine isolates harbored $q n r B$ and 3 isolates $q n r S$ genes. In all but three ciprofloxacin-resistant isolates, amino acid substitutions were detected in the QRDR of gyrA and/or parC. The genes sul1, sul2 and tet(A) were the most prevalent non- $\beta$-lactamase genes and non-PMQR detected.

The most dominant phylogenetic group was B1 $(n=10)$, followed by C $(n=6), \mathrm{B} 2(n=3)$, A and Clade II $(n=2)$ and singleton F. In two isolates the phylogroup could not be determined (Table 1$)$. E. coli clonotyping revealed 18 fumC and fimH $(\mathrm{CH})$ types obtained after DNA sequencing of these housekeeping genes. The most prevalent were CH11-0 and CH11-54 $(n=4)$, wherefrom five selected isolates belonged to three different STs (ST744, ST1287 and ST1642). Other prevalent CH types were CH65-32 ( $n=3)$; thereof one selected isolate belonged to ST469, CH40-30 $(n=2)$, represented by ST131 and $\mathrm{CH} 4-27(n=2)$ with one isolate belonging to ST648. The remaining clonotypes were singletons CH4-61, CH4-31, CH6-35, CH10-58, CH11-23, CH40-22, CH41-86, CH65-58, CH95-31, CH95-60, CH99-54, CH1430-142 and CH675-61 (Table 1, Figure 1). E. coli serogenotypes (somatic O and flagellar $\mathrm{H}$ antigens) were clearly determined in 8 isolates. MLST of bla $a_{\mathrm{NDM}-1}$ positive Enterobacter cloacae revealed that this isolate belonged to ST114 (Table 2). 
Table 1. Molecular characterization and resistance to antibiotics of canine and feline E. coli isolated from urogenital infections and originating from Austria (AUT) and Serbia (SRB).

\begin{tabular}{|c|c|c|c|c|c|c|c|c|c|c|c|c|c|c|c|c|}
\hline \multirow[b]{2}{*}{ Isolate } & \multirow[b]{2}{*}{ Country } & \multirow[b]{2}{*}{ Host } & \multirow[b]{2}{*}{ Source } & \multirow[b]{2}{*}{ Species } & \multirow[b]{2}{*}{ Phylogroup } & \multicolumn{2}{|c|}{ Serogenotyping } & \multicolumn{2}{|c|}{ Clonotype } & \multirow[t]{2}{*}{$\mathrm{ST}^{*}$} & \multicolumn{2}{|c|}{ Resistance Profile } & \multicolumn{3}{|c|}{ Mutation } & \multirow[b]{2}{*}{$\begin{array}{l}\text { Previous } \\
\text { therapy ** }\end{array}$} \\
\hline & & & & & & $\mathbf{O}$ & $\mathbf{H}$ & fumC & $f i m H$ & & Phenotype ** & Genotype & $\begin{array}{c}a m p C \\
\text { promoter }\end{array}$ & gyrA & parC & \\
\hline MDR56 & $\mathrm{SRB}$ & $\operatorname{dog}$ & $\begin{array}{l}\text { vaginal } \\
\text { swab }\end{array}$ & E. coli & A & 128 & 26 & 11 & 23 & n.t. & $\begin{array}{l}\text { AMP, CIP, } \\
\text { CHL, SXT }\end{array}$ & $\begin{array}{l}b l a_{\mathrm{TEM}}, c a t A 1, \\
\text { tet(B), } \operatorname{dfr} A 17\end{array}$ & & $\begin{array}{l}\text { Ser83Leu, } \\
\text { Asp87Asn }\end{array}$ & Ser80Ile & $\mathrm{AMC}, \mathrm{ENO}$ \\
\hline MDR63 & SRB & $\operatorname{dog}$ & urine & E. coli & $\mathrm{C}$ & 9 & 9 & 11 & 0 & n.t. & $\begin{array}{c}\text { AMP, CAZ, } \\
\text { CTX, CIP, TET, } \\
\text { CHL, SXT }\end{array}$ & $\begin{array}{c}\text { bla }_{\mathrm{TEM}}, \text { bla } \\
\text { strA, strB, } \\
\text { cat tet }(\mathrm{B}) \\
\text { dfr }, \text { sul1, sul2, } \\
\text { dfrA17, dfrA19 }\end{array}$ & w.t. & $\begin{array}{l}\text { Ser83Leu, } \\
\text { Asp87Asn }\end{array}$ & Ser80Ile & not known \\
\hline 193 & AUT & $\operatorname{dog}$ & bladder & E. coli & B1 & n.d. & 16 & 65 & 32 & n.t. & $\begin{array}{c}\text { AMP, CAZ, } \\
\text { CTX, CIP, TOB, } \\
\text { TET, SXT }\end{array}$ & $\begin{array}{c}\text { bla }_{\mathrm{TEM}}, \text { bla }_{\mathrm{CMY}}, \\
\text { qnrB, aac }(6) \\
\text { aac }(6)-\mathrm{I} b, \text { strB, } \\
\text { tet(A), sul1, sul } 2 \\
\text { dfrA5 }\end{array}$ & $-18,-1,+58$ & $\begin{array}{l}\text { Ser83Leu, } \\
\text { Asp87Asn }\end{array}$ & Ser80Ile & not known \\
\hline 2304 & AUT & $\operatorname{dog}$ & urine & E. coli & B1 & n.d. & 23 & 675 & 61 & n.t. & $\begin{array}{l}\text { AMP, CAZ, } \\
\text { CTX, CIP }\end{array}$ & $b l a_{\mathrm{TEM}}, b l a_{\mathrm{CMY}}$ & $-18,-1,+58$ & $\begin{array}{l}\text { Ser83Leu, } \\
\text { Asp87Asn }\end{array}$ & Ser80Ile & $\mathrm{CIP}$ \\
\hline 3056 & AUT & $\operatorname{dog}$ & urine & E. coli & unknown & 25 & 4 & 40 & 30 & n.t. & $\begin{array}{c}\text { AMP, CAZ, } \\
\text { CTX, ATM, CIP, } \\
\text { GEN, TOB, TET }\end{array}$ & $\begin{array}{c}b l a_{\mathrm{CTX}-\mathrm{M}-1} \\
\text { bla } \\
\text { aac (6)-Ib, tet }(\mathrm{A}) \\
\text { catB3 }\end{array}$ & & $\begin{array}{l}\text { Ser83Leu, } \\
\text { Asp87Asn }\end{array}$ & $\begin{array}{l}\text { Ser80Ile, } \\
\text { Glu84Val }\end{array}$ & $\begin{array}{c}\text { AMC, } \\
\text { Cephalosporin }\end{array}$ \\
\hline 3168 & AUT & $\operatorname{dog}$ & urine & E. coli & B1 & n.d. & 16 & 65 & 32 & n.t. & $\begin{array}{c}\text { AMP, CAZ, } \\
\text { CTX, CIP, TOB, } \\
\text { TET, SXT }\end{array}$ & $\begin{array}{c}b^{b_{\mathrm{TEM}},} \text { bla }_{\mathrm{CMY}} \\
q n r B, \text { aac6Ib } \\
\text { tet(A), sul1, sul2, } \\
\text { dfrA5 }\end{array}$ & $-18,-1,+58$ & $\begin{array}{l}\text { Ser83Leu, } \\
\text { Asp87Asn }\end{array}$ & Ser80Ile & $\begin{array}{l}\text { AMC, MAR, } \\
\text { CVN }\end{array}$ \\
\hline 2016 & AUT & $\operatorname{dog}$ & urine & E. coli & B1 & n.d. & 8 & 41 & 86 & n.t. & $\begin{array}{c}\text { AMP, CTX, FEP, } \\
\text { ATM, TET }\end{array}$ & 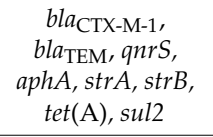 & & & & $\mathrm{ENO}, \mathrm{CVN}$ \\
\hline 2058 & AUT & $\operatorname{dog}$ & urine & E. coli & unknown & 25 & 4 & 40 & 22 & n.t. & $\begin{array}{c}\text { AMP, CTX, } \\
\text { ATM, TET, CHL }\end{array}$ & $\begin{array}{c}\text { bla }_{\mathrm{CTX}-\mathrm{M}-1} \\
\text { bla } \\
\text { stEM, str } A, \\
\text { strB, tet(A), floR }\end{array}$ & & & & none \\
\hline 4125 & AUT & $\operatorname{dog}$ & urine & E. coli & B1 & n.d. & 16 & 65 & 58 & n.t. & $\begin{array}{c}\text { AMP, CAZ, } \\
\text { CTX, CIP, TOB, } \\
\text { TET, SXT }\end{array}$ & $\begin{array}{c}\text { bla }_{\mathrm{TEM}}, \text { bla }_{\mathrm{CMY}}, \\
\text { qnrB, aac(6), } \\
\text { aac (6)-Ib, strA, } \\
\text { strB, tet (A), sul1, } \\
\text { sul2, dfrA5 }\end{array}$ & $-18,-1,+58$ & $\begin{array}{l}\text { Ser83Leu, } \\
\text { Asp87Asn }\end{array}$ & Ser80Ile & CHL \\
\hline
\end{tabular}


Table 1. Cont

\begin{tabular}{|c|c|c|c|c|c|c|c|c|c|c|c|c|c|c|c|c|}
\hline \multirow[b]{2}{*}{ Isolate } & \multirow[b]{2}{*}{ Country } & \multirow[b]{2}{*}{ Host } & \multirow[b]{2}{*}{ Source } & \multirow[b]{2}{*}{ Species } & \multirow[b]{2}{*}{ Phylogroup } & \multicolumn{2}{|c|}{ Serogenotyping } & \multicolumn{2}{|c|}{ Clonotype } & \multirow[t]{2}{*}{$\mathrm{ST}^{*}$} & \multicolumn{2}{|c|}{ Resistance Profile } & \multicolumn{3}{|c|}{ Mutation } & \multirow[b]{2}{*}{$\begin{array}{c}\text { Previous } \\
\text { therapy ** }\end{array}$} \\
\hline & & & & & & $\mathbf{O}$ & $\mathbf{H}$ & fumC & fimH & & Phenotype ** & Genotype & $\begin{array}{c}a m p C \\
\text { promoter }\end{array}$ & gyrA & parC & \\
\hline 1286 & AUT & cat & urine & E. coli & clade II & n.d. & n.d. & 4 & 27 & n.t. & $\begin{array}{c}\text { AMP, CAZ, } \\
\text { CTX, ATM, CIP, } \\
\text { TOB, TET, SXT }\end{array}$ & $\begin{array}{c}b^{b l a_{\mathrm{CMY}}}, \\
b l a_{\mathrm{OXA}-1}, b l a_{\mathrm{TEM}}, \\
a a c(6), \text { aac }(6)-\mathrm{Ib}, \\
\text { aadA4, strB, } \\
\text { catB3, tet( } \mathrm{A}), \\
\text { sul2, dfrA17 }\end{array}$ & +58 & $\begin{array}{l}\text { Ser83Leu, } \\
\text { Asp87Asn }\end{array}$ & Ser80Ile & none \\
\hline 2269 & AUT & $\operatorname{dog}$ & ejaculate & E. coli & $\mathrm{C}$ & n.d. & 30 & 99 & 54 & n.t. & $\begin{array}{l}\text { AMP, CIP, } \\
\text { GEN, TOB }\end{array}$ & $\begin{array}{l}b l a_{\mathrm{OXA}-1}, a a c(6) \\
a a c(6)-I b, c a t B 3\end{array}$ & & $\begin{array}{l}\text { Ser83Leu, } \\
\text { Asp87Asn }\end{array}$ & w.t. & none \\
\hline 2736 & AUT & $\operatorname{dog}$ & urin & E. coli & B1 & n.d. & 23 & 4 & 61 & n.t. & AMP, CIP, TET & $\operatorname{tet}(\mathrm{A})$ & & $\begin{array}{l}\text { Ser83Leu, } \\
\text { Asp87Asn }\end{array}$ & Ser80Ile & ENO \\
\hline 3441 & AUT & $\operatorname{dog}$ & urin & E. coli & C & n.d. & 9 & 4 & 31 & 744 & $\begin{array}{c}\text { AMP, CAZ, } \\
\text { CTX, CIP, TET, } \\
\text { CHL, SXT }\end{array}$ & 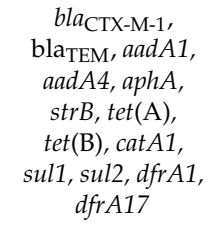 & & Ser83Leu & Ser80Ile & $\mathrm{CHL}$ \\
\hline 3448 & AUT & cat & urin & E. coli & $\mathrm{C}$ & n.d. & 9 & 11 & 0 & 744 & $\begin{array}{c}\text { AMP, CAZ, } \\
\text { CTX, ATM, CIP, } \\
\text { TET, CHL, SXT }\end{array}$ & $\begin{array}{c}\text { bla }_{\mathrm{CTX}-\mathrm{M}-1,}, \\
\text { bla } a_{\mathrm{TEM}}, \text { aadA2 } \\
\text { aadA4, aphA, } \\
\text { strA, strB, tet }(\mathrm{A}), \\
\text { tet }(\mathrm{B}), \operatorname{cat} A 1, \\
\text { sul1, sul2, dfrA1, } \\
\text { dfrA17 }\end{array}$ & & $\begin{array}{l}\text { Ser83Leu, } \\
\text { Asp87Asn }\end{array}$ & Ser80Ile & $\mathrm{CHL}$ \\
\hline 3534 & AUT & $\operatorname{dog}$ & urin & E. coli & B1 & n.d. & 9 & 11 & 54 & 744 & $\begin{array}{c}\text { AMP, CAZ, } \\
\text { CTX, CIP, GEN, } \\
\text { TOB, TET, } \\
\text { CHL, SXT }\end{array}$ & $\begin{array}{c}\text { bla } a_{\mathrm{TEM}}, \text { aad } A 1, \\
\text { aph } A, \text { str } A, \text { str } B \\
\operatorname{tet}(\mathrm{B}), \text { cat } A 1 \\
\text { flo } R, \text { sul } 2, d f r A 1\end{array}$ & & Ser83Leu & Ser80Ile & none \\
\hline 482 & AUT & $\operatorname{dog}$ & vagina & E. coli & C & n.d. & 9 & 1430 & 142 & n.t. & $\begin{array}{c}\text { AMP, CAZ, } \\
\text { CTX, ATM, CIP }\end{array}$ & 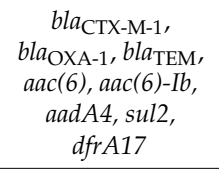 & & $\begin{array}{l}\text { Ser83Leu, } \\
\text { Asp87Asn }\end{array}$ & Ser80Ile & not known \\
\hline 4 & AUT & $\operatorname{dog}$ & urine & E. coli & clade II & n.d. & 6 & 10 & 58 & n.t. & $\begin{array}{c}\text { AMP, CTX, } \\
\text { ATM, CIP, GEN, } \\
\text { TOB, TET, CHL }\end{array}$ & $\begin{array}{c}\text { bla }_{\text {CTX-M-9, }} \\
\text { bla } \\
\text { Tet }(\mathrm{B}), \text { suld } \\
\text { dfrA17 } \\
\text { df }\end{array}$ & +58 & $\begin{array}{l}\text { Ser83Leu, } \\
\text { Asp87Asn }\end{array}$ & $\begin{array}{l}\text { Ser80Ile, } \\
\text { Glu84Gly }\end{array}$ & $\mathrm{CVN}$ \\
\hline
\end{tabular}


Table 1. Cont

\begin{tabular}{|c|c|c|c|c|c|c|c|c|c|c|c|c|c|c|c|c|}
\hline \multirow[b]{2}{*}{ Isolate } & \multirow[b]{2}{*}{ Country } & \multirow[b]{2}{*}{ Host } & \multirow[b]{2}{*}{ Source } & \multirow[b]{2}{*}{ Species } & \multirow[b]{2}{*}{ Phylogroup } & \multicolumn{2}{|c|}{ Serogenotyping } & \multicolumn{2}{|c|}{ Clonotype } & \multirow[t]{2}{*}{$\mathrm{ST}^{*}$} & \multicolumn{2}{|c|}{ Resistance Profile } & \multicolumn{3}{|c|}{ Mutation } & \multirow[b]{2}{*}{$\begin{array}{l}\text { Previous } \\
\text { therapy ** }\end{array}$} \\
\hline & & & & & & $\mathbf{O}$ & $\mathbf{H}$ & fumC & fimH & & Phenotype ${ }^{* *}$ & Genotype & $\begin{array}{c}a m p C \\
\text { promoter }\end{array}$ & gyrA & parC & \\
\hline 855 & AUT & $\operatorname{dog}$ & $\begin{array}{l}\text { vaginal } \\
\text { swab }\end{array}$ & E. coli & C & 9 & 9 & 11 & 54 & 1287 & $\begin{array}{l}\text { AMP, CTX, CIP, } \\
\text { TET, CHL, SXT }\end{array}$ & $\begin{array}{c}\text { bla }_{\mathrm{TEM}}, \text { bla } \\
\text { aad } A 1, \text { strB, } \\
\text { tet(B), cat } A 1 \\
\text { sul2, dfrA17 }\end{array}$ & w.t. & $\begin{array}{l}\text { Ser83Leu, } \\
\text { Asp87Asn }\end{array}$ & Ser80Ile & none \\
\hline 555 & AUT & $\operatorname{dog}$ & $\begin{array}{l}\text { prostata } \\
\text { cyst }\end{array}$ & E. coli & B2 & $25 b$ & 4 & 40 & 30 & 131 & $\begin{array}{c}\text { AMP, CAZ, } \\
\text { CTX, FEP, CIP, } \\
\text { GEN, TOB, } \\
\text { TET, SXT }\end{array}$ & $\begin{array}{c}\text { bla }_{\mathrm{CTX}-\mathrm{M}-15,} \\
\text { bla } a_{\mathrm{OXA} \mathrm{A}-1} \text {, aac }(6), \\
\text { aac(6)-Ib, aadA1, } \\
\text { aadA2, aadA4, } \\
\text { aadA5, tet(A), } \\
\text { catB3, sul1, sul2, } \\
\text { dfrA } 17\end{array}$ & & $\begin{array}{l}\text { Ser83Leu, } \\
\text { Asp87Asn }\end{array}$ & $\begin{array}{l}\text { Ser80Ile, } \\
\text { Glu84Gly }\end{array}$ & none \\
\hline 260 & AUT & $\operatorname{dog}$ & $\begin{array}{l}\text { vaginal } \\
\text { swab }\end{array}$ & E. coli & $\mathrm{F}$ & n.d. & 6 & 4 & 27 & 648 & $\begin{array}{c}\text { AMP, CAZ, } \\
\text { CTX, FEP, CIP, } \\
\text { GEN, TOB, TET, } \\
\text { CHL, SXT }\end{array}$ & $\begin{array}{c}\text { bla }_{\mathrm{CTX}-\mathrm{M}-15,} \\
\text { bla } \\
\text { aad }(6)-\mathrm{Ib} \text {, aad } 1, \\
\text { aadA2, tet( } \mathrm{A}), \\
\text { catB3, sul1, sul2 }\end{array}$ & & $\begin{array}{l}\text { Ser83Leu, } \\
\text { Asp87Asn }\end{array}$ & Ser80Ile & not knowr \\
\hline 655 & AUT & $\operatorname{dog}$ & urine & E. coli & B1 & n.d. & 7 & 4 & 31 & n.t. & $\begin{array}{c}\text { AMP, CAZ, } \\
\text { CTX, ATM, CIP, } \\
\text { TOB, TET, SXT }\end{array}$ & $\begin{array}{l}\text { bla } a_{\mathrm{TEM}}, b l a_{\mathrm{CMY}} \\
\text { aphA, strB, tet(B), } \\
\text { sul2 }\end{array}$ & w.t. & $\begin{array}{l}\text { Ser83Leu, } \\
\text { Asp87Asn }\end{array}$ & Ser80Ile & LEX \\
\hline 728 & AUT & $\operatorname{dog}$ & $\begin{array}{l}\text { vaginal } \\
\text { swab }\end{array}$ & E. coli & B1 & n.d. & ND & 65 & 32 & 469 & $\begin{array}{l}\text { AMP, CIP, TET, } \\
\text { CHL, SXT }\end{array}$ & $\begin{array}{l}\text { qnrS, tet(B), sul2, } \\
\text { sul3, dfr12, straA }\end{array}$ & & $\begin{array}{l}\text { Ser83Leu, } \\
\text { Asp87Asn }\end{array}$ & Ser80Ile & none \\
\hline 2189 & AUT & cat & urin & E. coli & B1 & 79 & 7 & 95 & 31 & 1463 & $\begin{array}{l}\text { AMP, CIP, TET, } \\
\text { CHL }\end{array}$ & $\begin{array}{l}\text { bla } \\
\text { cat } A 1\end{array}$ & & w.t. & Ser80Ile & not knowr \\
\hline 3512 & AUT & $\operatorname{dog}$ & $\begin{array}{l}\text { vaginal } \\
\text { swab }\end{array}$ & E. coli & B1 & n.d. & 7 & 11 & 54 & 1642 & $\begin{array}{l}\text { AMP, CIP, GEN, } \\
\text { TOB, CHL }\end{array}$ & $\begin{array}{l}\text { bla }_{\mathrm{TEM}}, \text { aad } A 4, \\
\text { aphA, strB, tet(B), } \\
\text { sul1, sul2, dfrA17 }\end{array}$ & & $\begin{array}{l}\text { Ser83Leu, } \\
\text { Asp87Asn }\end{array}$ & w.t. & none \\
\hline $2 \mathrm{srb}$ & SRB & $\operatorname{dog}$ & urin & E. coli & B1 & n.d. & 10 & 6 & 35 & n.t. & $\begin{array}{l}\text { AMP, CIP, } \\
\text { TET, SXT }\end{array}$ & $\begin{array}{c}\text { bla }{ }_{\mathrm{TEM}}, \text { aadA1, } \\
\text { strA, tet(A), sul1, } \\
\text { sul2, dfrA7, } \\
\text { dfrA17 }\end{array}$ & $-18,-1,+58$ & $\begin{array}{l}\text { Ser83Leu, } \\
\text { Asp87Asn }\end{array}$ & Ser80Ile & not knowr \\
\hline $1 \mathrm{srb}$ & SRB & $\operatorname{dog}$ & urin & E. coli & C & n.d. & 16 & 11 & 0 & n.t. & $\begin{array}{c}\text { AMP, CIP, GEN, } \\
\text { TET, SXT }\end{array}$ & $\begin{array}{c}\text { bla }_{\mathrm{TEM}}, \text { bla } \\
\text { aadA4 } \\
\text { tet(A) } \text {, strA, str } \text {, sul1, sul2, } \\
\text { dfrA17 }\end{array}$ & & $\begin{array}{l}\text { Ser83Leu, } \\
\text { Asp87Asn }\end{array}$ & Ser80Ile & not knowr \\
\hline
\end{tabular}


Table 1. Cont.

\begin{tabular}{|c|c|c|c|c|c|c|c|c|c|c|c|c|c|c|c|c|}
\hline \multirow[b]{2}{*}{ Isolate } & \multirow[b]{2}{*}{ Country } & \multirow[b]{2}{*}{ Host } & \multirow[b]{2}{*}{ Source } & \multirow[b]{2}{*}{ Species } & \multirow[b]{2}{*}{ Phylogroup } & \multicolumn{2}{|c|}{ Serogenotyping } & \multicolumn{2}{|c|}{ Clonotype } & \multirow[t]{2}{*}{$\mathrm{ST}^{*}$} & \multicolumn{2}{|c|}{ Resistance Profile } & \multicolumn{3}{|c|}{ Mutation } & \multirow[b]{2}{*}{$\begin{array}{c}\text { Previous } \\
\text { therapy }\end{array}$} \\
\hline & & & & & & $\mathrm{O}$ & $\mathbf{H}$ & fumC & fimH & & Phenotype ** & Genotype & $\begin{array}{c}a m p C \\
\text { promoter }\end{array}$ & gyrA & parC & \\
\hline ESBL1 & SRB & cat & $\begin{array}{l}\text { vaginal } \\
\text { swab }\end{array}$ & E. coli & C & n.d. & n.d. & 11 & 0 & n.t. & $\begin{array}{c}\text { AMP, CAZ, } \\
\text { CTX, ATM, CIP, } \\
\text { TOB, TET, SXT }\end{array}$ & 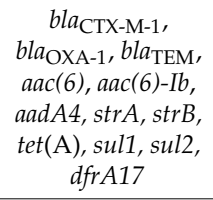 & & $\begin{array}{l}\text { Ser83Leu, } \\
\text { Asp87Asn }\end{array}$ & Ser80Ile & $\mathrm{AMC}$ \\
\hline ESBL41 & SRB & $\operatorname{dog}$ & $\begin{array}{l}\text { vaginal } \\
\text { swab }\end{array}$ & E. coli & A & n.d. & n.d. & 95 & 60 & n.t. & $\begin{array}{c}\text { AMP, CAZ, } \\
\text { CTX, CIP, GEN, } \\
\text { TOB, TET, } \\
\text { CHL, SXT }\end{array}$ & $\begin{array}{c}\text { bla }_{\mathrm{CTX}}-\mathrm{M}-1, \\
\text { bla } \\
\text { aad } A 2, \text { aph } A, \\
\text { str } A, \text { strB, tet }(\mathrm{A}), \\
\text { sul } 1, \text { sul } 2, \text { dfrA14 }\end{array}$ & & $\begin{array}{l}\text { Ser83Leu, } \\
\text { Asp87Asn }\end{array}$ & Ser80Ile & AMC \\
\hline
\end{tabular}

* sequence type. ** AMP-ampicillin, AMC-amoxicillin/clavulanate, LEX-cefalexin, CTX-cefotaxime, CAZ-ceftazidime, CVN-cefovecin, MEM-meropenem, ATM-aztreonam, FEP-cefepime, CIP-ciprofloxacin, ENO-enrofloxacin, MAR-marbofloxacin, GEN-gentamicin, TOB-tobramycin, TET-tetracycline, CHL-chloramphenicol, SXT- trimethoprim/sulfamethoxazole, NIT-nitrofurantoin, FOF-fosfomycin.

Table 2. Molecular characterization and resistance to antibiotics of canine and feline non-E. coli Enterobacterales isolated from urogenital infections and originating from Austria (AUT) and Serbia (SRB).

\begin{tabular}{|c|c|c|c|c|c|c|c|c|c|c|}
\hline \multirow[b]{2}{*}{ Isolate } & \multirow[b]{2}{*}{ Country } & \multirow[b]{2}{*}{ Host } & \multirow[b]{2}{*}{ Source } & \multirow[b]{2}{*}{ Species } & \multirow[b]{2}{*}{$\mathrm{ST}^{*}$} & \multicolumn{2}{|c|}{ Resistance Profile } & \multicolumn{2}{|c|}{ Mutation } & \multirow[b]{2}{*}{$\begin{array}{c}\text { Previous } \\
\text { therapy ** }\end{array}$} \\
\hline & & & & & & Phenotype ** & Genotype & gyrA & parC & \\
\hline $3 s r b$ & SRB & $\operatorname{dog}$ & $\begin{array}{l}\text { vaginal } \\
\text { swab }\end{array}$ & Serratia marcescens & n.t. & $\begin{array}{l}\text { AMP, CTX, TET, } \\
\text { CHL, SXT }\end{array}$ & $\begin{array}{c}\text { bla } a_{\mathrm{TEM}}, \text { aad } A 4, \\
\text { aph } A, \operatorname{str} B, \text { tet }(\mathrm{B}), \\
\text { cat } A 1\end{array}$ & & & not known \\
\hline 1919 & AUT & $\operatorname{dog}$ & urine & $\begin{array}{l}\text { Klebsiella } \\
\text { pneumoniae }\end{array}$ & n.t. & $\begin{array}{l}\text { AMP, CTX, CIP, } \\
\text { GEN, TOB, TET }\end{array}$ & $\begin{array}{c}b l a_{\mathrm{CTX}-\mathrm{M}-1} \\
\text { bla } a_{\mathrm{OXA}-1}, q n r B \\
\operatorname{aac}(6), \text { aac }(6)-\mathrm{Ib} \\
\text { catB3 }\end{array}$ & Ser83Ile & $\begin{array}{l}\text { codon 79Asp } \\
\text { silent } \\
\text { mutation } \\
(\mathrm{GAT}>\mathrm{GAC})\end{array}$ & not known \\
\hline 3938 & AUT & $\operatorname{dog}$ & urin & $\begin{array}{c}\text { Klebsiella } \\
\text { pneumoniae }\end{array}$ & n.t. & $\begin{array}{l}\text { AMP, CAZ, CTX, } \\
\text { CIP, GEN, TOB, } \\
\text { TET, CHL, SXT }\end{array}$ & $\begin{array}{c}\text { bla } a_{\mathrm{OXA}-1}, b l a_{\mathrm{SHV}}, \\
\text { aac }(6), a a c(6)-I b, \\
\text { qrnB, qnrS, aphA, } \\
\text { catB3, sul1 }\end{array}$ & w.t. & Ser80Ile & not known \\
\hline
\end{tabular}


Table 2. Cont.

\begin{tabular}{|c|c|c|c|c|c|c|c|c|c|c|}
\hline \multirow[b]{2}{*}{ Isolate } & \multirow[b]{2}{*}{ Country } & \multirow[b]{2}{*}{ Host } & \multirow[b]{2}{*}{ Source } & \multirow[b]{2}{*}{ Species } & \multirow[b]{2}{*}{$\mathrm{ST}$ * } & \multicolumn{2}{|c|}{ Resistance Profile } & \multicolumn{2}{|c|}{ Mutation } & \multirow[b]{2}{*}{$\begin{array}{l}\text { Previous } \\
\text { therapy ** }\end{array}$} \\
\hline & & & & & & Phenotype ** & Genotype & gyrA & $\operatorname{parC}$ & \\
\hline 3824 & AUT & $\operatorname{dog}$ & $\begin{array}{l}\text { prostata } \\
\text { swab }\end{array}$ & $\begin{array}{l}\text { Klebsiella } \\
\text { pneumoniae }\end{array}$ & n.t. & $\begin{array}{l}\text { AMP, CAZ, CTX, } \\
\text { FEP, CIP, TOB, } \\
\text { SXT, NIT }\end{array}$ & $\begin{array}{c}\text { bla }_{\mathrm{OXA}-1}, b l a_{\mathrm{SHV}} \\
\operatorname{aac}(6), \text { aac }(6)-I b \\
\text { aph } A, \text { qrnB, catB3, } \\
\text { sul1, dfrA12 }\end{array}$ & Asp87Asn & $\begin{array}{l}\text { Ser80Ile, } \\
\text { Glu84Gly }\end{array}$ & AMC, MAR \\
\hline 1049 & AUT & cat & urin & $\begin{array}{c}\text { Raoultella } \\
\text { ornithinolytica }\end{array}$ & n.t. & $\begin{array}{l}\text { AMP, CAZ, CTX, } \\
\text { CIP, GEN, TOB, } \\
\text { TET, CHL, SXT }\end{array}$ & $\begin{array}{l}\text { bla } a_{\mathrm{TEM}}, \text { aad } A 1, \\
\text { aph } A, \text { tet }(B), \text { sul1, } \\
\text { cat } A 1, \text { sul2 }\end{array}$ & w.t. & w.t. & not known \\
\hline 210 & AUT & $\operatorname{dog}$ & urine & Proteus mirabilis & n.t. & $\begin{array}{l}\text { AMP, CTX, FEP, } \\
\text { CHL, SXT }\end{array}$ & $\begin{array}{c}\text { bla }{ }_{\mathrm{TEM}}, \text { aad } A 1, \\
\text { aphA, strA, strB, } \\
\text { cat } A 1, \text { sul2, dfrA1 }\end{array}$ & & & none \\
\hline 3362 & AUT & cat & bladder wall & $\begin{array}{l}\text { Citrobacter } \\
\text { portucalensis }\end{array}$ & n.t. & $\begin{array}{c}\text { AMP, CAZ, CTX, } \\
\text { ATM, FEP, } \\
\text { CIP, SXT }\end{array}$ & $\begin{array}{c}b l a_{\mathrm{CTX}-\mathrm{M}-1}, b l a_{\mathrm{CMY}} \\
\text { qrnB, strA, strB } \\
\text { sul } 2, \text { dfr } A 1\end{array}$ & Ser83Leu & w.t. & MAR \\
\hline ESBL40 & SRB & Dog & $\begin{array}{l}\text { vaginal } \\
\text { swab }\end{array}$ & Enterobacter cloacae & 114 & $\begin{array}{c}\text { AMP, CAZ, CTX, } \\
\text { MEM, ATM, FEP, } \\
\text { CIP, TOB, TET, } \\
\text { CHL, SXT, FOF }\end{array}$ & 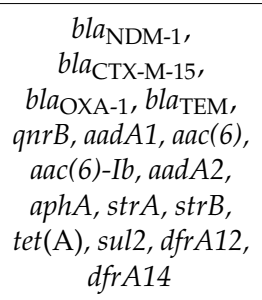 & Ser83Leu & w.t. & $\mathrm{AMC}, \mathrm{ENO}$ \\
\hline
\end{tabular}

* sequence type. ** AMP-ampicillin, AMC-amoxicillin/clavulanate, LEX-cefalexin, CTX-cefotaxime, CAZ-ceftazidime, CVN-cefovecin, MEM-meropenem, ATM-aztreonam, FEP-cefepime,

CIP-ciprofloxacin, ENO-enrofloxacin, MAR-marbofloxacin, GEN-gentamicin, TOB-tobramycin, TET-tetracycline, CHL-chloramphenicol, SXT- trimethoprim/sulfamethoxazole,

NIT-nitrofurantoin, FOF-fosfomycin. 


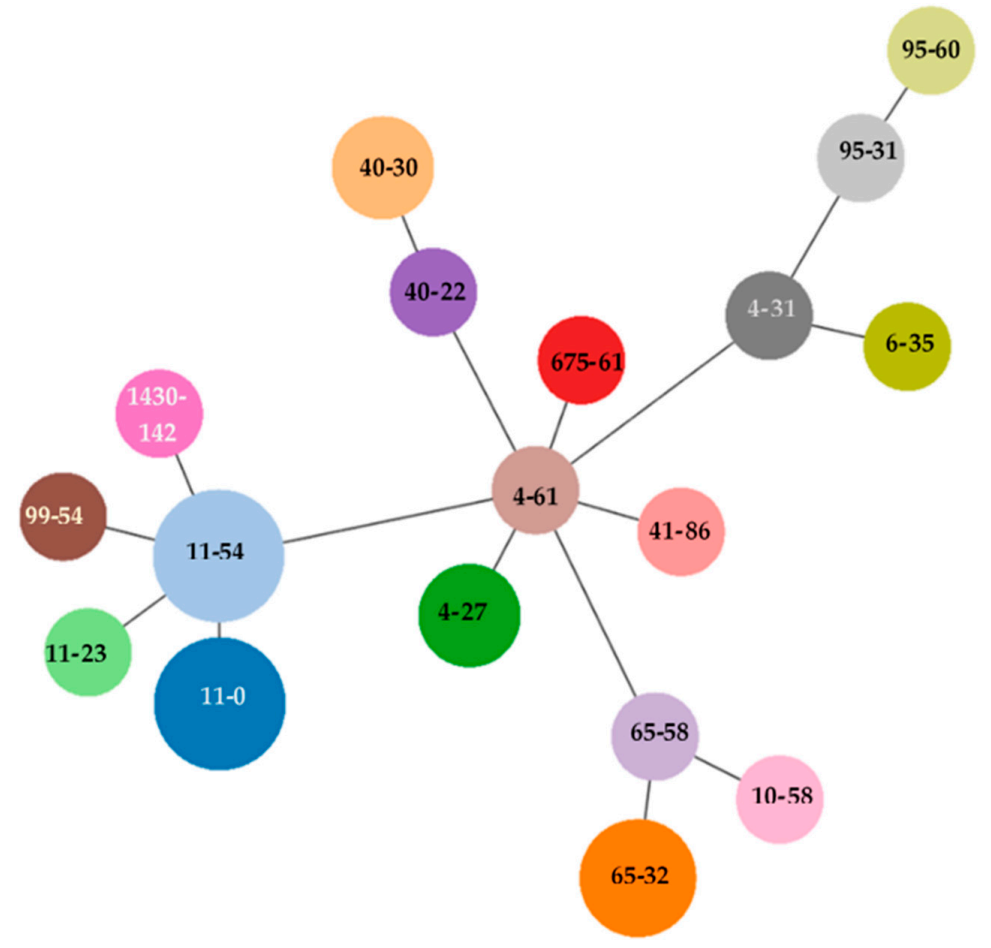

Figure 1. goeBURST diagram for the $\mathrm{CH}$ clonotyping data set (CHs in parenthesis) of 28 E. coli isolates. An eBURST diagram was calculated using PHYLOViZ with the goeBURST algorithm. E. coli isolates were grouped according to their $\mathrm{CH}$ profiles.

In twenty-five isolates at least two VFs associated with ExPEC/UPEC were found. The largest number within a total of seven VFs associated with ExPEC/UPEC was detected in two CH40-30 clonotypes that belonged to the $\mathrm{B} 2$ phylogroup, carried bla $a_{\mathrm{CTX}-\mathrm{M}-15}$ and harbored $p a p \mathrm{C}, f i m \mathrm{H}$, iuc $\mathrm{D}$, iha, $n f a$, sat, iss and pap $\mathrm{C}$, fim $\mathrm{H}, i u c \mathrm{D}$, sat, cnf1, iss, iha, respectively. Both isolates carried the $\mathrm{H} 4$ gene and allele-specific PCR confirmed the presence of the $r f b \mathrm{O} 25 b$ locus of ST131 but also in an CF40-22 isolate (Table 3).

Table 3. Virulence factors detected in E. coli isolates obtained from dogs and cats with urogenital infections and originating from Austria (AUT) and Serbia (SRB).

\begin{tabular}{|c|c|c|c|c|c|}
\hline Isolate & Country & Host & Source & Species & Virulence Factors \\
\hline MDR56 & SRB & $\operatorname{dog}$ & vaginal swab & E. coli & tsh, mchF, hemL, intl1, iroN, iss, fimH, iucD \\
\hline MDR63 & SRB & $\operatorname{dog}$ & urin & E. coli & $\operatorname{sen} B$, hemL, intl1, iss, iucD \\
\hline 193 & AUT & $\operatorname{dog}$ & bladder & E. coli & $m c h F$, hemL, intl1, iroN, iss \\
\hline 2304 & AUT & $\operatorname{dog}$ & urine & E. coli & lpfA, mchF, hemL, iroN, iss, fimH \\
\hline 3056 & AUT & $\operatorname{dog}$ & urine & E. coli & iha, prfB, cnf1, sat, iss. fimH, iucD, papC \\
\hline 3168 & AUT & $\operatorname{dog}$ & urine & E. coli & hemL, iss, fimH, iucD \\
\hline 2016 & AUT & $\operatorname{dog}$ & urine & E. coli & $\operatorname{lpfA}, \mathrm{cma}$, hemL, iroN, iss, fimH \\
\hline 2058 & AUT & $\operatorname{dog}$ & urine & E. coli & prfB, $m c h F$, iroN, iss, fimH, iucD, papC \\
\hline 4125 & AUT & $\operatorname{dog}$ & urine & E. coli & lpfA, $m c h F$, hemL, intl1, iroN, iss, fimH, iucD \\
\hline 1286 & AUT & cat & urine & E. coli & $\operatorname{prfB}$, hemL, iss, fimH, papC \\
\hline 2269 & AUT & $\operatorname{dog}$ & ejaculate & E. coli & hemL, fimH, iucD \\
\hline 2736 & AUT & $\operatorname{dog}$ & urin & E. coli & $\operatorname{lpfA}$, hemL, fim $H$ \\
\hline 3441 & AUT & $\operatorname{dog}$ & urin & E. coli & tsh, cba, cma, mchF, hemL, intl1, iron, iss, fimH, iucD \\
\hline 3448 & AUT & cat & urin & E. coli & tsh, cba, cma, mchF, hemL, intl1, iron, iss, fim H, iucD \\
\hline 3534 & AUT & $\operatorname{dog}$ & urin & E. coli & $\operatorname{prfB}, m c m A$, hemL, intl2, ire $A$, fim $H, i u c D$ \\
\hline 482 & AUT & $\operatorname{dog}$ & vagina & E. coli & hemL, fimH \\
\hline 4 & AUT & $\operatorname{dog}$ & urine & E. coli & hemL, iss, fimH \\
\hline 855 & AUT & $\operatorname{dog}$ & vaginal swab & E. coli & hemL, intl1, iroN, iss, iucD \\
\hline 555 & AUT & $\operatorname{dog}$ & prostata cyst & E. coli & iha, nfaE, prfB, sat, senB, intl1, iss, fimh, iucD, papC \\
\hline
\end{tabular}


Table 3. Cont.

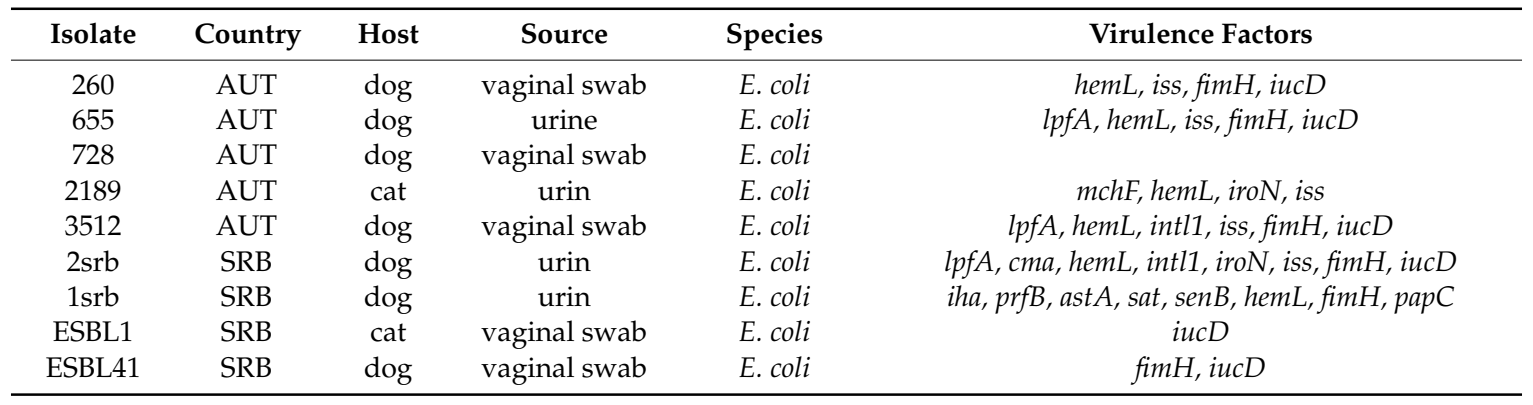

\section{Discussion}

Among Austrian E. coli isolates, clones belonging to B2 (phylotype)-O25b:H4 (serotype)-ST131 (sequence type)-CH40-30 (clonotype)-CTX-M-15 and FQR isolated from a canine prostate specimen and E. coli F-ONT(non-typeable):H6-ST648-CH4-27-CTX-M-15 (and FQR resistant) isolated from a vaginal swab of a dog were the most significant. The same applies to NDM-1 positive Enterobacter cloacae from Serbia. E. coli O25b:H4-ST131 (CH40-30 [CTX-M-15]) is widely recognized as one of the most important pandemic pathogens. This clone has been shown to cause various infections in humans and animals ranging from asymptomatic bacteriuria to complicated UGIs, septicemia, meningitis, and pneumonia. In several publications E. coli ST131 has been unanimously classified as ExPEC and/or UPEC $[1,2,4,7,8,11,12,14,15,17,35,38-40]$. According to origin and site of infection, E. coli ST131 strains were additionally categorized as FUPEC, NEMEC, APEC or SEPEC. It is believed that the global dissemination of E. coli ST131 as well as E. coli ST648 has contributed to the worldwide emergence of CTX-M-15 producing E. coli [14,41]. Anyway, CTX-M-15 is a mostly plasmid-transferable gene and not all ST131 E. coli isolates harbor it. Thus, E. coli ST131 may be susceptible to cephalosporins [42]. ST131 is mostly (but not necessarily) associated with fluoroquinolone resistance [35,43]. So far, CTX-M-15 harboring and fluoroquinolone-resistant E. coli B2-O25b:H4-ST131 has been described as the cause of UGI in dogs in Portugal, the Netherlands, Switzerland, the United Kingdom and France [24,33]. It has also been isolated from dogs and cats in the USA, from dogs, cats and horses in Denmark, Germany and Spain and from dogs in China $[35,44]$. E. coli B2-O25b:H4-ST131-CTX-M-27 variant has been isolated from urine specimen of dogs and cats in Japan [31]. Non CTX-M-15, fluoroquinolone-resistant E. coli B2-O25b:H4-ST131 has also been detected in the urinary tract of dogs and cats in the USA and its transmission from companion animals to humans has been suspected suggesting dogs and cats as possible reservoir of this clone [14]. However, E. coli ST131 has never been reported to be isolated from the prostate of a dog, as was the case in our study. Zoonotic transmission of E. coli ST131 is still the subject of scientific debates. Transmission of E. coli ST131 from poultry to humans and rats has been observed and transfer of E. coli ST131 via meat products, particularly from chicken meat to humans causing UGI has been proven $[2,13,17]$. In Austria, this pandemic clone has only been isolated from wildlife so far [45].

Another clone, E. coli F (D)-ST648 (CTX-M-15) which also has readily been classified as ExPEC or/and UPEC was recognized as the zoonotic pandemic agent of various types of infections most common in humans but also in livestock and companion animals [40]. It has been detected in pigs, calves and other livestock in Germany and the Netherlands, dogs and cats in the USA, Germany and Italy as well as in wild birds in Mongolia and Germany [34]. It was first isolated as a cause of cystitis in a cat in 2013 in Switzerland [15], later it has been detected in urine samples of dogs with UGI, also in Switzerland [33]. It was a frequently isolated clone (D-ST648 [CMY, TEM]) from intestinal samples originating from stray dogs reported in a study from South Korea [23]. Another significant finding in this study is, to our knowledge the first isolation of a CTX-M-15 and NDM-1, together with OXA 1, TEM producing Enterobacter cloacae ST114 from a vaginal swab of a dog from Serbia (strain ESBL40). Carbapenems-resistance in bacteria of animal origin is a subject of great concern due to their 
importance in therapy of life-threatening infections in human and in veterinary medicine $[26,43,46]$. Although several publications state that the risk of carbapenem resistance in animals is low due to the limited use of this class in veterinary medicine $[26,43,46]$, this is the second report on the detection of carbapenemases carrying bacteria associated with canine UGI from Serbia [36] and $b l a_{\mathrm{NDM}}, b l a_{\mathrm{VIM}}$, $b l a_{\mathrm{IMP}}, b l a_{\mathrm{OXA}}$ and $b l a_{\mathrm{KPC}}$ carbapenemases have been detected worldwide in companion animals. Unlike carbapenemases, CTX-M-15 is highly prevalent in Enterobacter cloacae and CTX-M-15-producing ST114 Enterobacter cloacae has been recently reported as a high-risk clone for public health which was predominant in cats, dogs and horses $[47,48]$.

Of the other ST found in our study, E. coli ST744 was represented by three isolates (B1-ONT:H9ST744-CH11-54 [TEM]; C-ONT:H9-ST744-CH11-0 [CTX-M-1, TEM]; C-ONT:H9-ST744-CH4-31 [CTX-M-1, TEM]). This ST has previously been isolated from urine samples of dogs (carrying CTX-M-1, CTX-M-15, CTX-M-14) [33] and E. coli ST744-CTX-M-1 was also reported in livestock [43].

In our study, one AmpC/ESBL E. coli C-O9:H9-ST1287-CH11-54 (TEM, CMY) was detected in a vaginal swab of a dog. Fluoroquinolone resistant, non-AmpC, non-ESBL E. coli ST1287 categorized as APEC has been reported in a parrot pet from Brazil [49] and in a blood sample from a patient with septicemia as well as in the feces of a healthy individual [21,50]. This ST seems to be rare and, to our knowledge, AmpC/ESBL/FQR E. coli ST1287 has been isolated from a dog for the first time.

E. coli B1-ST469 and B1-ST1463, which have also been found in our study, have rarely been reported, mostly in humans and turkey [22]. To the best of our knowledge, these two STs have not been isolated from cases of UGI in companion animals, so far.

One E. coli isolated from the vagina of a dog has been typed as B1-ONT:H7-ST1642-CH11-54 (TEM). The same ST (B1-O8:H7-ST1642) harboring CTX-M-55 has been isolated from a urine specimen of a cat in Japan [31], the CTX-M-14 harboring variant has been detected in human blood samples [40], the CTX-M-15 variant has been found in feces of healthy lambs in Brazil [30] and the E. coli B1-ST1642 harboring $b l a_{\mathrm{TEM}}$ and $b l a_{\mathrm{CMY}}$ has been detected in an intestinal specimen from a stray dog in South Korea [23].

In CTX-M harboring isolates, bla $a_{\mathrm{CTX}-\mathrm{M}-1}$ was more common which is also inconsistent with findings in other studies mostly reporting CTX-M-15 being predominant among ESBL producing enterobacteria isolated from humans and animals [24]. In addition, CTX-M-14 and CTX-M-55 harboring Enterobacterales have been increasingly reported in companion animals [23,24], which were

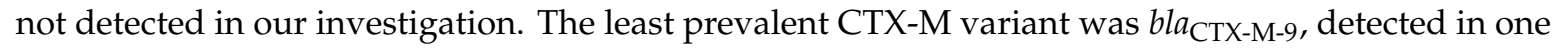
AmpC/ESBL/FQR E. coli from Austria (ID 4) (Clade II-CH10-58 [CTX-M-9, TEM]). In one large study among companion animals in China, out of 107 CTX-M producing E. coli, only one was CTX-M-9 [51]. In another study from UK, out of 876 investigated and 164 cephalosporin-resistant E. coli originating from companion animals, neither one harbored CTX-M-9 [24].

Based on the published surveys [19,26-28], the use of critically important antimicrobials (amongst them 3rd and 4th generation cephalosporins and fluoroquinolones) is cited as being frequently prescribed for dogs and cats in a number of countries including Belgium, Spain, France, the United Kingdom and Germany. Carbapenems are mostly prescribed in complicated cases (MDR infections) based on results of susceptibility testing and treatment durations are longer than typically recommended in human medicine [26].

In our study, MDR (ESBL/FQR) E. coli ST131 (555) and Klebsiella pneumoniae (3824) have been isolated from two canine prostate samples. Not all antibiotics are capable of penetrating the prostate tissue, so the therapy choice is initially limited to enrofloxacin, trimethoprim and fosfomycin [18]. Both prostate isolates from our study were susceptible only to fosfomycin which has not been licensed for the use in companion animals, neither in Serbia, nor in Austria. Therefore, this antibiotic is not included in routine susceptibility testing, though out of label use would be feasible. Besides, CLSI does not provide interpretative categories for fosfomycin in its document [29] and human-related interpretative categories may be used, although interpretation should then be performed with caution. The finding of MDR bacteria in the canine prostate (including human-related clones such as E. coli 
ST131) is becoming a reality and one should expect more such cases in the future. Besides, out of 36 MDR strains included in this study, 35 were susceptible to fosfomycin. The only fosfomycin resistant strain was a carbapenem resistant Enterobacter cloacae ST114 isolated from the vagina of a Serbian dog. Since fosfomycin is a critically important human-related antibiotic according to WHO [52], the emergence of bacterial strains susceptible only to fosfomycin in companion animals may lead to an undesired, more frequent use of this antibiotic in veterinary medicine in the future.

Ten E. coli and the Citrobacter portucalensis isolate (9 originating from urine samples) from our study exhibited an ESBL/AmpC/FQR resistance phenotype and all were susceptible to nitrofurantoin and fosfomycin. Nitrofurantoin has also been listed in the ISCAID for the treatment of MDR related UGIs in companion animals. Interpretative categories for nitrofurantoin are presented in the CLSI VET01, Ed4E document, though nitrofurantoin has not been licensed for use in companion animals, neither in Austria nor in Serbia.

Although it has been proven that ExPEC/UPEC clones that share the same antibiotic resistance patterns may cause UGIs in humans as well as in animals, recommendations for the treatment of UGIs in humans differ significantly from guidelines related to animals. The Infectious Diseases Society of America (IDSA) in collaboration with the European Society for Microbiology and Infectious Diseases (ESCMID) removed beta-lactams and fluoroquinolones from the list of first-line antibiotics for the treatment of uncomplicated UGIs in adult humans [53]. Instead, nitrofurantoin, trimethoprim-sulfamethoxazole and fosfomycin are recommended. Amoxicillin and ampicillin have been removed because of their relatively poor efficacy and the high prevalence of antimicrobial resistance to these agents worldwide [53].

There are no defined criteria for strict categorization of E. coli strain as ExPEC or UPEC or any other subcategory (APEC, NEMEC, SEPEC), because all these subcategories mostly share the same virulence genes $[1,4,7-9,13,17,38,39,54-56]$. Some authors assume that the presence of at least two of the following genetic determinants indicates categorization of an isolate as ExPEC/UPEC: papA and/or papC (P fimbriae structural subunit and assembly), sfa/focDE (S and F1C fimbriae), afa/draBC (Dr binding adhesins), iutA (aerobactin system) and kpsM II (group 2 capsule) [1]. This minimal predictive set of ExPEC virulence genes has not been thoroughly validated and thus is considered incomplete as strains meeting this definition do not always cause disease. Accordingly, drawbacks of such a scheme were shown in a study [57] in which E. coli ST648 has been categorized as pandemic ExPEC but results demonstrated that out of 47 ST648 isolates only 17 were assignable to ExPEC based on the aforementioned scheme. Thus, we did not use this scheme in our study. However, since we considered iucD and iutA to have the same function [40] encoding aerobactin ferric receptors, 3 of our investigated isolates that were shown to harbor $p a p \mathrm{C} / i u c \mathrm{D}$ could potentially be classified as ExPEC/UPEC. Those were isolate 555 (B2-O25b:H4-ST131-CH40-30 [CTX-M-15, OXA1]) and further two isolates which belonged to the cam fumC cluster 2058 (O25:H4CH40-22 [CTX-M-1, TEM]) and 3056 (O25:H4CH40-30 [CTX-M-1, OXA-1]) probably sharing the same ST.

Another approach is to associate virulence factor(s) (VF(s)) with ST, CH types, phylogroups, antibiotic resistance and thus to establish pathogenic and zoonotic potential of the suspected ExPECs. Therefore, we included VFs mostly associated with ExPEC or UPEC [17] amongst them papC, fim H, $i u c \mathrm{D}$, sat, $c n f 1$, iss, iha, ast, $n f a$, iro $\mathrm{N}$, tsh and $h l y$. Based on this expanded list of VFs our 18 isolates investigated can be assigned to ExPEC/UPEC (containing 3 or more VFs from the expanded VF list).

The fim $\mathrm{H}$ gene was found in $61 \%(n=20$ Austrian and $n=2$ Serbian) isolates. It encodes FimH adhesins/type 1 fimbriae, which are essential virulence factors for uropathogenic E. coli and their successful colonization of the urinary bladder epithelium [58] but are also referred as a major virulence factor of E. coli belonging to the ExPEC category [5,6,58]. Anyway, this type of adhesin is not only typical for uropathogenic but also for intestinal as well as enterohaemorrhagic (EHEC) isolates. Reports show that fim $\mathrm{H}$ is equally represented in E. coli isolated from both humans and animals [59].

The gene $i u c \mathrm{D}$ encodes for an enzyme included in the aerobactin biosynthesis pathway [60]. The aerobactin siderophore system is an important virulence factor and it is absent in avirulent 
E. coli strains, thus, finding $i u c \mathrm{D}$ in $55 \%$ ( $n=15$ Austrian, $n=5$ Serbian) may serve as evidence for pathogenicity of the isolates [60]. The secreted autotransporter toxin SAT is a virulence factor of UPEC and/or diffusely adhering E. coli (DAEC) both associated with pyelonephritis and urosepsis [61]. Sat is reported to be predominant in E. coli of human origin [57]. In our study, it was found in 3 isolates, two from Austria (555: B2-O25b:H4-ST131-CH40-30; 3056: O25:H4-CH40-30) and one from Serbia (1srb: C-CH11-0 [TEM, OXA-2]). The latter also harbored the ast gene encoding heat-stable enterotoxin 1 which is mostly found in ETEC, thus isolate 1srb probably belonged to diarrheal E. coli.

$P$ fimbriae (pap) are the second common virulence factor of UPEC, which plays an important role in the pathogenesis of ascending UGIs and pyelonephritis in humans [61], it was found in $13.8 \%(n=5)$ isolates in our study.

Gene tsh encoding temperature-sensitive haemagglutinin was found in two Austrian (3441: C-ONT:H9-ST744; 3448: C-ST744) and one Serbian strain (MDR56: A-O128:H26). It is predominant in E. coli isolated from animals [57] and mostly present in APECs as previously reported with a prevalence of more than 50\% in APEC, $4.5 \%$ in UPEC and $11.5 \%$ in NMEC isolates tested [5]. Strain MDR56 C-STNT probably was of intestinal origin. This is supported by the finding that it belongs to phylogroup A to which commensal E. coli from the gastrointestinal tract mostly belong [38]. Besides, MDR56 was typed as $\mathrm{O} 128$ (H26) which is a serotype often reported as shiga toxin-producing E. coli (STEC) mostly found in sheep and humans [59]; however, MDR56 was shown to be negative for stx.

The increased serum survival gene iss has been reported in E. coli isolated from humans and animals [57] and in approximately $60 \%$ of UPEC and NMEC strains. It has also been associated with APEC strains [60]. In our study it was found in $55 \%(n=20)$ of isolates.

\section{Materials and Methods}

During the study period between October 2010 and March 2015, 2398 samples from Austria as well as between January 2012 and March 2015, 144 samples from Serbia taken from the canine and feline urogenital tract were considered in the present study. If the microbiological examination yielded bacterial growth, isolates were identified to the species level by matrix-assisted laser desorption/ionization-time of flight (MALDI-TOF) mass spectrometry (Bruker Daltonik, Heidelberg, Germany). Antimicrobial susceptibility testing was performed by agar disk-diffusion according to standards of the Clinical and Laboratory Standards Institute (CLSI) [62]. Escherichia coli ATCC ${ }^{\circledR} 25922$ served as quality control strain. The following antimicrobials were used-ampicillin, cefotaxime, ceftazidime, cefepime, aztreonam, meropenem, gentamicin, amikacin, tobramycin, ciprofloxacin, trimethoprim-sulfamethoxazole, tetracycline fosfomycin, nitrofurantoin and chloramphenicol (Becton Dickinson, Heidelberg, Germany). In addition, isolates were checked for extended-spectrum $\beta$-lactamase (ESBL) production by ESBL-test via agar disk diffusion combination disk tests using cefotaxime and ceftazidime with and without clavulanic acid [62]. Furthermore, cefoxitin (30 $\mu \mathrm{g})$ was added to this test, to detect AmpC phenotypes. Isolates belonging to Enterobacterales and were broad-spectrum cephalosporin-resistant and/or fluoroquinolone-resistant were included in the present study.

All isolates were obtained from samples during routine bacteriological diagnostics at the Institute of Microbiology, University of Veterinary Medicine, Vienna, Austria as well as the Department for Microbiology, Faculty of Veterinary Medicine University of Belgrade, Serbia. All these clinical samples were received from third parties and therefore not subject to reporting obligations of the Ethics and Animal Welfare Commission of the University of Veterinary Medicine in Vienna and not subjected to Ethics Commission for the experimental animals welfare protection of the Faculty of Veterinary Medicine in Belgrade. All isolates were stored at $-80^{\circ} \mathrm{C}$ until further examination.

The serogenotyping and the detection of resistance genes and major virulence genes of E. coli isolates was performed using the miniaturized microarray-based E. coli PanType AS-2 kit (Alere, Jena, Germany). The same assay was used to detect resistant determinants in non-E. coli Enterobacterales. Resistance genes in one carbapenem-resistant Enterobacter cloacae-complex isolate were analyzed by 
CarbDetect-AS-2 Kit microarray (Alere, Jena, Germany) [63,64]. PCRs were performed to detect tet(A) and tet(B) in carbapenem resistant Enterobacter cloacae as well as to amplify $b l a_{\mathrm{NDM}}$ and $b l a_{\mathrm{CTX}}$ which were then sequenced $[65,66]$. Sequences were aligned with BLAST (https://blast.ncbi.nlm.nih.gov/Blast.cgi) and compared with reference sequences available in GenBank and the National Center for Biotechnology Information (NCBI) database (http://www.ncbi.nlm.nih.gov/pathogens/beta-lactamase-data-resources/). The quinolone resistance-determining regions (QRDR) of gyr $A$ and parC in ciprofloxacin-resistant isolates were amplified by PCR and sequenced [67]. E. coli isolates displaying the AmpC phenotype were also analyzed for mutations in the chromosomal ampC promoter/attenuator region as described previously [68]. Allele-specific PCR was performed to identify the O25 clone of selected E. coli isolates [69]. The E. coli isolate was assigned to a phylogroup using the quadruplex assignment method [70]. Clonal relatedness of E. coli isolates was assessed by two-locus sequence typing of combined data of fum $\mathrm{C}$ and fim $\mathrm{H}$ sequences as described by Weissman et al. [71]. Allele and CF clonotype numbers were used for goeBURST analysis using PHYLOViZ [72]. Selected E. coli isolates were further subjected to multilocus sequence typing (MLST) [73]. Allelic profiles and sequence types (ST) were determined by querying the E. coli MLST website (http://enterobase.warwick.ac.uk/species/ecoli/allele_st_search). The carbapenem-resistant Enterobacter cloacae isolate was characterized by MLST according to the protocol recommended by Miyoshi-Akiyama et al. [74] and ST was determined using Enterobacter cloacae MLST database (https: //pubmlst.org/ecloacae/).

\section{Conclusions}

The study discovered E. coli B2-O25b:H4-ST131-CH40-30-CTX-M-15 and FQR in a prostate specimen from a dog and E. coli F (D)-ONT:H6-ST648-CH4-27-CTX-M-15 and FQR in a canine vaginal sample, which are important pandemic pathogens that have been shown to cause various infections in humans and animals. Furthermore, carbapenem-resistant Enterobacter cloacae ST114 harboring bla $a_{\mathrm{NDM}}$, $b l a_{\mathrm{CTX}-\mathrm{M}-15}, b l a_{\mathrm{OXA}-1}$ and $b l a_{\mathrm{TEM}}$ was detected in a canine vaginal swab from Serbia, which is considered to be the first report of NDM-1 harboring Enterobacter cloacae isolated from a dog. The findings in this study suggest a possible reverse zoonotic transmission of ExPEC/UPEC—companion animals may be infected by human-related clones because of their close co-existence and then may become an important source of those clones for the human population.

Author Contributions: Conceptualization, I.L., D.M, F.K., S.S.-S. and J.S.; methodology, I.L. and M.P.S.; validation, I.L., M.P.S., F.K. and S.S.-S.; formal analysis, I.L., D.M. and M.P.S.; investigation, I.L., D.M., F.K. and S.S.-S.; resources, J.S.; data curation, I.L.; writing-original draft preparation, I.L. and D.M.; writing-review and editing, I.L., D.M. and J.S. All authors have read and agreed to the published version of the manuscript.

Funding: This research received no external funding. Open Access Funding by the University of Veterinary Medicine Vienna.

Acknowledgments: We would also like to express our thanks to Michael Steinbrecher and Barbara Tischler for technical assistance and all students who contributed to this study. We are grateful to Ursula Schober for helping us to obtain the Vetmeduni Vienna fund for inviting international guests to participate in research activities.

Conflicts of Interest: The authors declare no conflict of interest.

\section{References}

1. Singer, R.S. Urinary Tract Infections Attributed to Diverse ExPEC Strains in Food Animals: Evidence and Data Gaps. Front. Microbiol. 2015, 6, 28. [CrossRef] [PubMed]

2. Nordstrom, L.; Liu, C.M.; Price, L.B. Foodborne Urinary Tract Infections: A New Paradigm for Antimicrobial-Resistant Foodborne Illness. Front. Microbiol. 2013, 4, 29. [CrossRef] [PubMed]

3. Qekwana, D.N.; Phophi, L.; Naidoo, V.; Oguttu, J.W.; Odoi, A. Antimicrobial Resistance among Escherichia coli Isolates from Dogs Presented with Urinary Tract Infections at a Veterinary Teaching Hospital in South Africa. BMC Vet. Res. 2018, 14, 288. [CrossRef] [PubMed] 
4. Sarowska, J.; Futoma-Koloch, B.; Jama-Kmiecik, A.; Frej-Madrzak, M.; Ksiazczyk, M.; Bugla-Ploskonska, G.; Choroszy-Krol, I. Virulence Factors, Prevalence and Potential Transmission of Extraintestinal Pathogenic Escherichia coli Isolated from Different Sources: Recent Reports. Gut Pathog. 2019, 11, 10. [CrossRef] [PubMed]

5. Antão, E.-M.; Wieler, L.H.; Ewers, C. Adhesive Threads of Extraintestinal Pathogenic Escherichia coli. Gut Pathog. 2009, 1, 22. [CrossRef]

6. Johnson, J.R.; Kuskowski, M.A.; Owens, K.; Gajewski, A.; Winokur, P.L. Phylogenetic Origin and Virulence Genotype in Relation to Resistance to Fluoroquinolones and/or Extended-Spectrum Cephalosporins and Cephamycins among Escherichia coli Isolates from Animals and Humans. J. Infect. Dis. 2003, 5, 759-768. [CrossRef]

7. Freitag, T.; Squires, R.A.; Schmid, J.; Elliott, J. Feline Uropathogenic Escherichia coli from Great Britain and New Zealand Have Dissimilar Virulence Factor Genotypes. Vet. Microbiol. 2005, 106, 79-86. [CrossRef]

8. Smith, J.L.; Fratamico, P.M.; Gunther, N.W. Extraintestinal Pathogenic Escherichia coli. Foodborne Pathog. Dis. 2007, 4, 134-163. [CrossRef]

9. Guignot, J.; Chaplais, C.; Coconnier-Polter, M.H.; Servin, A.L. The Secreted Autotransporter Toxin, Sat, Functions as a Virulence Factor in Afa/Dr Diffusely Adhering Escherichia coli by Promoting Lesions in Tight Junction of Polarized Epithelial Cells. Cell. Microbiol. 2007, 9, 204-221. [CrossRef]

10. Price, L.B.; Hungate, B.A.; Koch, B.J.; Davis, G.S.; Liu, C.M. Colonizing Opportunistic Pathogens (COPs): The Beasts in All of Us. PLoS Pathog. 2017, 13, e1006369. [CrossRef]

11. Liu, C.M.; Stegger, M.; Aziz, M.; Johnson, T.J.; Waits, K.; Nordstrom, L.; Gauld, L.; Weaver, B.; Rolland, D.; Statham, S.; et al. Escherichia coli ST131-H22 as a Foodborne Uropathogen. MBio 2018, 9, e00470-18. [CrossRef] [PubMed]

12. Shaik, S.; Ranjan, A.; Tiwari, S.K.; Hussain, A.; Nandanwar, N.; Kumar, N.; Jadhav, S.; Semmler, T.; Baddam, R.; Islam, M.A.; et al. Comparative Genomic Analysis of Globally Dominant ST131 Clone with Other Epidemiologically Successful. MBio 2017, 8, e01596-17. [CrossRef] [PubMed]

13. LeStrange, K.; Markland, S.M.; Hoover, D.G.; Sharma, M.; Kniel, K.E. An Evaluation of the Virulence and Adherence Properties of Avian Pathogenic Escherichia coli. One Heath 2017, 4, 22-26. [CrossRef] [PubMed]

14. Johnson, J.R.; Miller, S.; Johnston, B.; Clabots, C.; DebRoy, C. Sharing of Escherichia coli Sequence Type ST131 and Other Multidrug-Resistant and Urovirulent E. Coli Strains among Dogs and Cats within a Household. J. Clin. Microbiol. 2009, 47, 3721-3725. [CrossRef]

15. Huber, H.; Zweifel, C.; Wittenbrink, M.M.; Stephan, R. ESBL-Producing Uropathogenic Escherichia coli Isolated from Dogs and Cats in Switzerland. Vet. Microbiol. 2013, 162, 992-996. [CrossRef] [PubMed]

16. Madec, J.Y.; Haenni, M.; Nordmann, P.; Poirel, L. Extended-Spectrum $\beta$-Lactamase/AmpC- and Carbapenemase-Producing Enterobacteriaceae in Animals: A Threat for Humans? Clin. Microbiol. Infect. 2017, 23, 826-833. [CrossRef] [PubMed]

17. Johnson, T.J.; Wannemuehler, Y.; Johnson, S.J.; Stell, A.L.; Doetkott, C.; Johnson, J.R.; Kim, K.S.; Spanjaard, L.; Nolan, L.K. Comparison of Extraintestinal Pathogenic Escherichia coli Strains from Human and Avian Sources Reveals a Mixed Subset Representing Potential Zoonotic Pathogens. Appl. Environ. Microbiol. 2008, 74, 7043-7050. [CrossRef] [PubMed]

18. Weese, J.S.; Blondeau, J.; Boothe, D.; Guardabassi, L.G.; Gumley, N.; Papich, M.; Jessen, L.R.; Lappin, M.; Rankin, S.; Westropp, J.L.; et al. International Society for Companion Animal Infectious Diseases (ISCAID) Guidelines for the Diagnosis and Management of Bacterial Urinary Tract Infections in Dogs and Cats. Vet. J. 2019, 247, 8-25. [CrossRef]

19. Roberts, M.; White, J.; Lam, A. Prevalence of Bacteria and Changes in Trends in Antimicrobial Resistance of Escherichia coli Isolated from Positive Canine Urinary Samples from an Australian Referral Hospital over a 5-Year Period (2013-2017). Vet. Rec. Open 2019, 6, e000345. [CrossRef]

20. Marques, C.; Gama, L.T.; Belas, A.; Bergström, K.; Beurlet, S.; Briend-Marchal, A.; Broens, E.M.; Costa, M.; Criel, D.; Damborg, P.; et al. European Multicenter Study on Antimicrobial Resistance in Bacteria Isolated from Companion Animal Urinary Tract Infections. BMC Vet. Res. 2016, 12, 213. [CrossRef]

21. Obeng-Nkrumah, N.; Labi, A.K.; Blankson, H.; Awuah-Mensah, G.; Oduro-Mensah, D.; Anum, J.; Teye, J.; Kwashie, S.D.; Bako, E.; Ayeh-Kumi, P.F.; et al. Household Cockroaches Carry CTX-M-15-, OXA-48- and NDM-1-Producing Enterobacteria, and Share Beta-Lactam Resistance Determinants with Humans. BMC Microbiol. 2019, 19, 272. [CrossRef] [PubMed] 
22. Pietsch, M.; Irrgang, A.; Roschanski, N.; Brenner, M.G.; Hamprecht, A.; Rieber, H.; Käsbohrer, A.; Schwarz, S.; Rösler, U.; Kreienbrock, L.; et al. Whole Genome Analyses of CMY-2-Producing Escherichia coli Isolates from Humans, Animals and Food in Germany. BMC Genom. 2018, 19, 601. [CrossRef] [PubMed]

23. Tamang, M.D.; Nam, H.M.; Jang, G.C.; Kim, S.R.; Chae, M.H.; Jung, S.C.; Byun, J.W.; Park, Y.H.; Lim, S.K. Molecular Characterization of Extended-Spectrum- $\beta$-Lactamase- Producing and Plasmid-Mediated AmpC $\beta$-Lactamase-Producing Escherichia coli Isolated from Stray Dogs in South Korea. Antimicrob. Agents Chemother. 2012, 56, 2705-2712. [CrossRef] [PubMed]

24. Bortolami, A.; Zendri, F.; Maciuca, E.I.; Wattret, A.; Ellis, C.; Schmidt, V.; Pinchbeck, G.; Timofte, D. Diversity, Virulence, and Clinical Significance of Extended-Spectrum $\beta$-Lactamase- And PAmpC-Producing Escherichia coli from Companion Animals. Front. Microbiol. 2019, 10, 1260. [CrossRef]

25. National Office for Animal Health UK. Critically Important Antibiotics in Veterinary Medicine: European Medicines Agency Recommendations. Available online: https://www.noah.co.uk/wp-content/ uploads/2016/12/NOAH-briefing-on-CIAs-07122016.pdf (accessed on 28 May 2020).

26. Smith, A.; Wayne, A.S.; Fellman, C.L.; Rosenbaum, M.H. Usage Patterns of Carbapenem Antimicrobials in Dogs and Cats at a Veterinary Tertiary Care Hospital. J. Vet. Intern. Med. 2019, 33, 1677-1685. [CrossRef]

27. Dupouy, V.; Abdelli, M.; Moyano, G.; Arpaillange, N.; Bibbal, D.; Cadiergues, M.C.; Lopez-Pulin, D.; Sayah-Jeanne, S.; De Gunzburg, J.; Saint-Lu, N.; et al. Prevalence of Beta-Lactam and Quinolone/Fluoroquinolone Resistance in Enterobacteriaceae from Dogs in France and Spain-Characterization of ESBL/PAmpC Isolates, Genes, and Conjugative Plasmids. Front. Vet. Sci. 2019, 30, 279. [CrossRef]

28. Singleton, D.A.; Sánchez-Vizcaíno, F.; Dawson, S.; Jones, P.H.; Noble, P.J.M.; Pinchbeck, G.L.; Williams, N.J.; Radford, A.D. Patterns of Antimicrobial Agent Prescription in a Sentinel Population of Canine and Feline Veterinary Practices in the United Kingdom. Vet. J. 2017, 224, 18-24. [CrossRef]

29. Clinical and Laboratory Standards Institute. Performance Standards for Antimicrobial Disk and Dilution Susceptibility Tests for Bacteria Isolated from Animals, 4th ed.; CLSI Supplement VET08; Clinical and Laboratory Standards Institute: Wayne, PA, USA, 2018.

30. Gozi, K.S.; Froes, J.R.; Ajude, L.P.; Da Silva, C.R.; Baptista, R.S.; Peiró, J.R.; Marinho, M.; Mendes, L.C.N.; Nogueira, M.C.L.; Casella, T. Dissemination of Multidrug-Resistant Commensal Escherichia coli in Feedlot Lambs in Southeastern Brazil. Front. Microbiol. 2019, 10, 1394. [CrossRef]

31. Harada, K.; Nakai, Y.; Kataoka, Y. Mechanisms of Resistance to Cephalosporin and Emergence of O25b-ST131 Clone Harboring CTX-M-27 $\beta$-Lactamase in Extraintestinal Pathogenic Escherichia coli from Dogs and Cats in Japan. Microbiol. Immunol. 2012, 56, 480-485. [CrossRef]

32. Maluta, R.P.; Logue, C.M.; Casas, M.R.T.; Meng, T.; Guastalli, E.A.L.; Rojas, T.C.G.; Montelli, A.C.; Sadatsune, T.; Ramos, M.D.C.; Nolan, L.K.; et al. Overlapped Sequence Types (STs) and Serogroups of Avian Pathogenic (APEC) and Human Extra-Intestinal Pathogenic (ExPEC) Escherichia coli Isolated in Brazil. PLoS ONE 2014, 9, e105016. [CrossRef] [PubMed]

33. Zogg, A.L.; Simmen, S.; Zurfluh, K.; Stephan, R.; Schmitt, S.N.; Nüesch-Inderbinen, M. High Prevalence of Extended-Spectrum $\beta$-Lactamase Producing Enterobacteriaceae among Clinical Isolates from Cats and Dogs Admitted to a Veterinary Hospital in Switzerland. Front. Vet. Sci. 2018, 5, 62. [CrossRef] [PubMed]

34. Wagner, S.; Gally, D.L.; Argyle, S.A. Multidrug-Resistant Escherichia coli from Canine Urinary Tract Infections Tend to Have Commensal Phylotypes, Lower Prevalence of Virulence Determinants and AmpC-Replicons. Vet. Microbiol. 2014, 169, 171-178. [CrossRef] [PubMed]

35. Nicolas-Chanoine, M.H.; Bertrand, X.; Madec, J.Y. Escherichia coli ST131, an Intriguing Clonal Group. Clin. Microbiol. Rev. 2014, 27, 543-754. [CrossRef]

36. Misic, D.; Asanin, J.; Spergser, J.; Szostak, M.; Loncaric, I. OXA-72-Mediated Carbapenem Resistance in Sequence Type 1 Multidrug Colistin-Resistant Acinetobacter baumannii Associated with Urinary Tract Infection in a Dog from Serbia. Antimicrob. Agents Chemother. 2018, 62, e00219-18. [CrossRef]

37. Sweeney, M.T.; Lubbers, B.V.; Schwarz, S.; Watts, J.L. Applying Definitions for Multidrug Resistance, Extensive Drug Resistance and Pandrug Resistance to Clinically Significant Livestock and Companion Animal Bacterial Pathogens. J. Antimicrob. Chemother. 2018, 73, 1460-1463. [CrossRef]

38. Khairy, R.M.; Mohamed, E.S.; Ghany, H.M.A.; Abdelrahim, S.S. Phylogenic Classification and Virulence Genes Profiles of Uropathogenic E. Coli and Diarrhegenic E. coli Strains Isolated from Community Acquired Infections. PLoS ONE 2019, 14, e0222441. [CrossRef] 
39. Müller, C.M.; Dobrindt, U.; Nagy, G.; Emödy, L.; Uhlin, B.E.; Hacker, J. Role of Histone-like Proteins H-NS and StpA in Expression of Virulence Determinants of Uropathogenic Escherichia coli. J. Bacteriol. 2006, 188, 5428-5438. [CrossRef]

40. Mamani, R.; Flament-Simon, S.C.; García, V.; Mora, A.; Alonso, M.P.; López, C.; García-Meniño, I.; Díaz-Jiménez, D.; Blanco, J.E.; Blanco, M.; et al. Sequence Types, Clonotypes, Serotypes, and Virotypes of Extended-Spectrum $\beta$-Lactamase-Producing Escherichia coli Causing Bacteraemia in a Spanish Hospital over a 12-Year Period (2000 to 2011). Front. Microbiol. 2019, 10, 1530. [CrossRef]

41. Pitout, J.D.D. Extraintestinal Pathogenic Escherichia coli: An Update on Antimicrobial Resistance, Laboratory Diagnosis and Treatment. Expert Rev. Anti-Infect. Ther. 2012, 10, 1165-1176. [CrossRef] [PubMed]

42. Boehmer, T.; Vogler, A.J.; Thomas, A.; Sauer, S.; Hergenroether, M.; Straubinger, R.K.; Birdsell, D.; Keim, P.; Sahl, J.W.; Williamson, C.H.D.; et al. Phenotypic Characterization and Whole Genome Analysis of Extended-Spectrum Betalactamase-Producing Bacteria Isolated from Dogs in Germany. PLoS ONE 2018, 13, e0206252. [CrossRef]

43. Poirel, L.; Madec, J.-Y.; Lupo, A.; Schink, A.-K.; Kieffer, N.; Nordmann, P.; Schwarz, S. Antimicrobial Resistance in Escherichia coli. In Antimicrobial Resistance in Bacteria from Livestock and Companion Animals; ASM Press: Washington, DC, USA, 2018; Volume 6. [CrossRef]

44. Pitout, J.D.D.; Nordmann, P.; Laupland, K.B.; Poirel, L. Emergence of Enterobacteriaceae Producing Extended-Spectrum $\beta$-Lactamases (ESBLs) in the Community. J. Antimicrob. Chemother. 2005, 56, 52-59. [CrossRef]

45. Loncaric, I.; Stalder, G.L.; Mehinagic, K.; Rosengarten, R.; Hoelzl, F.; Knauer, F.; Walzer, C. Comparison of ESBL-And AmpC Producing Enterobacteriaceae and Methicillin-Resistant Staphylococcus aureus (MRSA) Isolated from Migratory and Resident Population of Rooks (Corvus Frugilegus) in Austria. PLoS ONE 2013, 8, e84048. [CrossRef]

46. Köck, R.; Daniels-Haardt, I.; Becker, K.; Mellmann, A.; Friedrich, A.W.; Mevius, D.; Schwarz, S.; Jurke, A. Carbapenem-Resistant Enterobacteriaceae in Wildlife, Food-Producing, and Companion Animals: A Systematic Review. Clin. Microbiol. Infect. 2018, 24, 1241-1250. [CrossRef] [PubMed]

47. Desvars-Larrive, A.; Ruppitsch, W.; Lepuschitz, S.; Szostak, M.P.; Spergser, J.; Feßler, A.T.; Schwarz, S.; Monecke, S.; Ehricht, R.; Walzer, C.; et al. Urban Brown Rats (Rattus Norvegicus) as Possible Source of Multidrug-Resistant Enterobacteriaceae and Meticillin-Resistant Staphylococcus Spp., Vienna, Austria, 2016 and 2017. Eurosurveillance 2019, 24, 1900149. [CrossRef] [PubMed]

48. Haenni, M.; Saras, E.; Ponsin, C.; Dahmen, S.; Petitjean, M.; Hocquet, D.; Madec, J.Y. High Prevalence of International ESBL CTX-M-15-Producing Enterobacter cloacae ST114 Clone in Animals. J. Antimicrob. Chemother. 2016, 71, 1497-1500. [CrossRef] [PubMed]

49. Borges, C.A.; Beraldo, L.G.; Maluta, R.P.; Cardozo, M.V.; Barboza, K.B.; Guastalli, E.A.L.; Kariyawasam, S.; DebRoy, C.; Ávila, F.A. Multidrug-Resistant Pathogenic Escherichia coli Isolated from Wild Birds in a Veterinary Hospital. Avian Pathol. 2017, 46, 76-83. [CrossRef] [PubMed]

50. Ho, P.L.; Leung, L.M.; Chow, K.H.; Lai, E.L.; Lo, W.U.; Ng, T.K. Prevalence of Aminoglycoside Modifying Enzyme and 16S Ribosomal RNA Methylase Genes among Aminoglycoside-Resistant Escherichia coli Isolates. J. Microbiol. Immunol. Infect. 2016, 49, 123-126. [CrossRef]

51. Sun, Y.; Zeng, Z.; Chen, S.; Ma, J.; He, L.; Liu, Y.; Deng, Y.; Lei, T.; Zhao, J.; Liu, J.H. High Prevalence of BlaCTX-M Extended-Spectrum $\beta$-Lactamase Genes in Escherichia coli Isolates from Pets and Emergence of CTX-M-64 in China. Clin. Microbiol. Infect. 2010, 16, 1475-1481. [CrossRef]

52. World Health Organization. Global Priority List of Antibiotic-Resistant Batceria to Guide Research, Discovery, and Development of New Antibiotics. 2017. Available online: http://www.who.int/medicines/publications/ WHO-PPL-Short_Summary_25Feb-ET_NM_WHO.pdf (accessed on 27 May 2020).

53. Johnson, J.R.; Sannes, M.R.; Croy, C.; Johnston, B.; Clabots, C.; Kuskowski, M.A.; Bender, J.; Smith, K.E.; Winokur, P.L.; Belongia, E.A. Antimicrobial Drug-Resistant Escherichia coli from Humans and Poultry Products, Minnesota and Wisconsin, 2002-2004. Emerg. Infect. Dis. 2007, 13, 838-846. [CrossRef]

54. Johnson, J.R.; Russo, T.A. Molecular Epidemiology of Extraintestinal Pathogenic (Uropathogenic) Escherichia coli. Int. J. Med. Microbiol. 2005, 295, 383-404. [CrossRef] 
55. Ewers, C.; Bethe, A.; Stamm, I.; Grobbel, M.; Kopp, P.A.; Guerra, B.; Stubbe, M.; Doi, Y.; Zong, Z.; Kola, A.; et al. CTX-M-15-D-ST648 Escherichia coli from Companion Animals and Horses: Another Pandemic Clone Combining Multiresistance and Extraintestinal Virulence? J. Antimicrob. Chemother. 2014, 69, 1224-1230. [CrossRef] [PubMed]

56. Bouckaert, J.; Mackenzie, J.; De Paz, J.L.; Chipwaza, B.; Choudhury, D.; Zavialov, A.; Mannerstedt, K.; Anderson, J.; Piérard, D.; Wyns, L.; et al. The Affinity of the FimH Fimbrial Adhesin Is Receptor-Driven and Quasi-Independent of Escherichia coli Pathotypes. Mol. Microbiol. 2006, 61, 1556-1568. [CrossRef] [PubMed]

57. Manges, A.R.; Harel, J.; Masson, L.; Edens, T.J.; Portt, A.; Reid-Smith, R.J.; Zhanel, G.G.; Kropinski, A.M.; Boerlin, P. Multilocus Sequence Typing and Virulence Gene Profiles Associated with Escherichia coli from Human and Animal Sources. Foodborne Pathog. Dis. 2015, 12, 302-310. [CrossRef] [PubMed]

58. Herrero, M.; De Lorenzo, V.; Neilands, J.B. Nucleotide Sequence of the IucD Gene of the PCoIV-K30 Aerobactin Operon and Topology of Its Product Studied with PhoA and LacZ Gene Fusions. J. Bacteriol. 1988, 170, 56-64. [CrossRef] [PubMed]

59. Brett, K.N.; Ramachandran, V.; Hornitzky, M.A.; Bettelheim, K.A.; Walker, M.J.; Djordjevic, S.P. Stx1c Is the Most Common Shiga Toxin 1 Subtype among Shiga Toxin-Producing Escherichia oli Isolates from Sheep but Not among Isolates from Cattle. J. Clin. Microbiol. 2003, 41, 926-936. [CrossRef] [PubMed]

60. Johnson, T.J.; Wannemuehler, Y.M.; Nolan, L.K. Evolution of the Iss Gene in Escherichia coli. Appl. Environ. Microbiol. 2008, 74, 2360-2369. [CrossRef]

61. Bien, J.; Sokolova, O.; Bozko, P. Role of Uropathogenic Escherichia coli Virulence Factors in Development of Urinary Tract Infection and Kidney Damage. Int. J. Nephrol. 2012, 2012, 681473. [CrossRef]

62. Clinical and Laboratory Standards Institute. Performance Standards for Antimicrobial Susceptibility Testing, 28th ed.; CLSI Supplement M100S; CLSI: Wayne, PA, USA, 2018.

63. Braun, S.D.; Monecke, S.; Thürmer, A.; Ruppelt, A.; Makarewicz, O.; Pletz, M.; Reißig, A.; Slickers, P.; Ehricht, R. Rapid Identification of Carbapenemase Genes in Gram-Negative Bacteria with an Oligonucleotide Microarray-Based Assay. PLoS ONE 2014, 9, e102232. [CrossRef] [PubMed]

64. Gwida, M.; Awad, A.; El-Ashker, M.; Hotzel, H.; Monecke, S.; Ehricht, R.; Müller, E.; Reißig, A.; Barth, S.A.; Berens, C.; et al. Microarray-Based Detection of Resistance and Virulence Factors in Commensal Escherichia coli from Livestock and Farmers in Egypt. Vet. Microbiol. 2020, 240, 108539. [CrossRef]

65. Loncaric, I.; Beiglböck, C.; Feßler, A.T.; Posautz, A.; Rosengarten, R.; Walzer, C.; Ehricht, R.; Monecke, S.; Schwarz, S.; Spergser, J.; et al. Characterization of ESBL- and AmpC-Producing and Fluoroquinolone-Resistant Enterobacteriaceae Isolated from Mouflons (Ovis Orientalis Musimon) in Austria and Germany. PLoS ONE 2016, 18, e0155786. [CrossRef]

66. Nordmann, P.; Poirel, L.; Carrër, A.; Toleman, M.A.; Walsh, T.R. How to Detect NDM-1 Producers. J. Clin. Microbiol. 2011, 49, 718-721. [CrossRef] [PubMed]

67. Everett, M.J.; Jin, Y.F.; Ricci, V.; Piddock, L.J.V. Contributions of Individual Mechanisms to Fluoroquinolone Resistance in 36 Escherichia coli Strains Isolated from Humans and Animals. Antimicrob. Agents Chemother. 1996, 40, 2380-2386. [CrossRef]

68. Caroff, N.; Espaze, E.; Bérard, I.; Richet, H.; Reynaud, A. Mutations in the AmpC Promoter of Escherichia coli Isolates Resistant to Oxyiminocephalosporins without Extended Spectrum $\beta$-Lactamase Production. FEMS Microbiol. Lett. 1999, 173, 459-465. [CrossRef]

69. Clermont, O.; Dhanji, H.; Upton, M.; Gibreel, T.; Fox, A.; Boyd, D.; Mulvey, M.R.; Nordmann, P.; Ruppé, E.; Sarthou, J.L.; et al. Rapid Detection of the O25b-ST131 Clone of Escherichia coli Encompassing the CTX-M-15-Producing Strains. J. Antimicrob. Chemother. 2009, 64, 274-277. [CrossRef] [PubMed]

70. Clermont, O.; Christenson, J.K.; Denamur, E.; Gordon, D.M. The Clermont Escherichia coli Phylo-Typing Method Revisited: Improvement of Specificity and Detection of New Phylo-Groups. Environ. Microbiol. Rep. 2013, 5, 58-65. [CrossRef] [PubMed]

71. Weissman, S.J.; Johnson, J.R.; Tchesnokova, V.; Billig, M.; Dykhuizen, D.; Riddell, K.; Rogers, P.; Qin, X.; Butler-Wu, S.; Cookson, B.T.; et al. High-Resolution Two-Locus Clonal Typing of Extraintestinal Pathogenic Escherichia coli. Appl. Environ. Microbiol. 2012, 78, 1353-1360. [CrossRef] [PubMed] 
72. Francisco, A.P.; Vaz, C.; Monteiro, P.T.; Melo-Cristino, J.; Ramirez, M.; Carriço, J.A. PHYLOViZ: Phylogenetic inference and data visualization for sequence based typing methods. BMC BioInform. 2012, 13, 87. [CrossRef]

73. Wirth, T.; Falush, D.; Lan, R.; Colles, F.; Mensa, P.; Wieler, L.H.; Karch, H.; Reeves, P.R.; Maiden, M.C.J.; Ochman, H.; et al. Sex and Virulence in Escherichia Coli: An Evolutionary Perspective. Mol. Microbiol. 2006, 60, 1136-1151. [CrossRef]

74. Miyoshi-Akiyama, T.; Hayakawa, K.; Ohmagari, N.; Shimojima, M.; Kirikae, T. Multilocus Sequence Typing (MLST) for Characterization of Enterobacter cloacae. PLoS ONE 2013, 8, e66358. [CrossRef] 